\title{
Retroauricular Robotic Thyroidectomy with Concomitant Neck-Lift Surgery
}

\author{
Salah Eldin Mohamed, MD', Ahmad Saeed, MD', Thomas Moulthrop, MD², and Emad Kandil, MD, FACS ${ }^{1}$ \\ ${ }^{1}$ Department of Surgery, Division of Endocrine and Oncologic Surgery, Tulane University School of Medicine, \\ New Orleans, LA; ${ }^{2}$ Division of Otolaryngology, Tulane University School of Medicine, New Orleans, LA
}

\begin{abstract}
Background. Robotic-assisted thyroid surgery using a retroauricular approach was reported as a novel remote access technique for hemithyroidectomy. We report our experience with this remote access technique using a single incision in the retroauricular crease and occipital hairline incision. For the first time, we show additional neck-lift surgery performed concomitantly to achieve better cosmetic outcomes.

Methods. Robotic retroauricular left hemithyroidectomy with concomitant neck-lift surgery was performed in a 59year-old female patient who was referred for management of a $1.7 \mathrm{~cm}$ thyroid nodule located in the mid-lower right thyroid lobe, with fine-needle aspiration biopsy suggestive for follicular neoplasm. The patient had redundant submental skin in her neck, and was planning for a future neck-lift surgery. A concomitant neck lift was performed by the plastic surgeon using the same retroauricular approach to perform the operation. The patient agreed to participate in Institutional Review Board approved protocol.
\end{abstract}

Results. Total operative time was 115 min-flap creation time was $50 \mathrm{~min}$, robot docking time was $10 \mathrm{~min}$, operative console time was $25 \mathrm{~min}$, and concomitant necklift surgery extended for $30 \mathrm{~min}$. Estimated blood loss was approximately $30 \mathrm{ml}$. The patient was discharged home on the same day of surgery and had no postoperative complications. Final pathology confirmed benign follicular adenoma.

Conclusions. Our experience with robotic retroauricular thyroidectomy showed that it is a feasible and safe remote access approach. We suggest that concomitant neck lift can be done in a select group of patients with excess skin on the neck. This approach can be offered to patients with benign and indeterminate thyroid lesions, and future prospective studies are warranted to evaluate the oncological efficacy of this approach in patients with thyroid cancer.

FINANCIAL DISCLOSURE This research work was fully supported by the Tulane University Medical Center. The authors have no financial interest in companies or other entities that have an interest in the information included in this contribution.

CONFLICTS OF INTEREST None.
Electronic supplementary material The online version of this article (doi:10.1245/s10434-013-3470-z) contains supplementary material, which is available to authorized users.
(C) Society of Surgical Oncology 2014

First Received: 27 November 2013;

Published Online: 14 January 2014

E. Kandil, MD, FACS

e-mail: Ekandil@tulane.edu 